\section{Une carte de santé pour la Suisse}

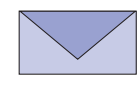

Dans un article paru dans le BMS, un certain M. Beng, ingénieur diplômé EPF, de Siemens Suisse SA, semble prendre les médecins pour des idiots [1]. En effet, il vante avec force les mérites des cartes de santé électroniques et les présente comme étant LA solution.

A ce propos, j'aimerais lui poser les questions suivantes:

- Qui assumera les coûts exorbitants de l'introduction et du traitement de ces cartes dans les centres de traitement?

- Est-on en mesure de garantir des standards uniformes supranationaux? (Dans la mesure où les documents sont rédigés dans nos caractères d'imprimerie, je peux lire les principales informations concernant les personnes qui arrivent chez nous, quelle que soit leur origine, ce qu'une carte de santé ne me permettra jamais de faire.)

- M. Beng s'est-il posé la question de savoir pourquoi les cartes au porteur posent souvent des problèmes au moment d'effectuer des paiements?

- S'est-il demandé ce qu'il adviendra dans 10 ou 20 ans des données saisies lorsqu'aucun appareil ne sera plus en mesure de les lire (en effet, tous les appareils électroniques sont dépassés après 5 ans et inutilisables au bout de 10 ans)? Les dossiers médicaux que je conserve depuis 35 ans me fournissent aujourd'hui encore des informations quant aux résultats d'examens et constatations médicales, ce que ni appareils électroniques ni microfilms ne peuvent faire après 15 ou 30 ans.

- Qui est responsable si un résultat important ou une ordonnance n'est pas enregistré sur la carte? Même des choses aussi évidentes que les groupes sanguins ne sont plus une banalité aujourd'hui au vu des techniques d'analyses actuelles, et les effets secondaires n'ont été démontrés, grâce au système CYP notamment, que ces dernières années.

1 Beng M. Eine Gesundheitskarte für die Schweiz. Schweiz Ärztezeitung 2005;86(37):2139-4.
Les dispositifs de sécurité et la protection des données sectorielles sont de toute façon illusoires sur une carte accessible à tout un chacun.

\section{Conclusion}

La carte de santé proposée est encore plus inutilisable dans la pratique que le code CIM-10 et va même jusqu'à mettre en danger la santé de son propriétaire. Au lieu de réaliser des économies, on créera des coûts supplémentaires importants si l'on introduit cette carte sur le plan national.

Dr J. Riediker, Effretikon

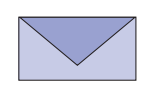

M. Beng a publié dans le BMS un article très informatif sur l'éventuelle introduction d'une carte de santé en Suisse. Les médecins praticiens sont cependant d'avis que quelques remarques s'imposent à ce sujet:

- L'article souligne notamment le grand potentiel d'une carte de santé dans le cadre d'une solution axée sur un serveur avec des dossiers électroniques de patients. Il signale toutefois également les coûts monstrueux (en milliards de francs) liés à ce type de solution. Et prend pour exemple l'introduction d'une carte de santé et d'un système de gestion des cartes en Lombardie, une région d'environ 9 millions d'habitants, par l'entreprise Siemens où l'auteur est employé. L'article a donc été rédigé par un expert de l'industrie mais les références concernant certaines affirmations-clés font malheureusement défaut.

- Si l'on effectue une recherche dans les archives électroniques du BMS au moyen des mots-clés «carte de santé» «carte de patient», «carte d'assuré», on trouvera 19 articles. Il s'agit surtout d'articles dans lesquels le motclé est mentionné uniquement dans le cadre d'autres considérations sur le thème des soins de santé par voie électronique («eHealthCare»). Certains de ces articles ont été rédigés par Martin Denz, l'ancien responsable de la FMH dans ce domaine. D'autres contributions ont été écrites par des techniciens, des spécialistes en santé publique ou par des économistes. Aucun article, excepté deux lettres de lecteur, n'a été rédigé par des confrères praticiens ou en collaboration avec eux. Depuis le départ de M. Denz, les médecins se taisent. Vu les bouleversements qui nous attendent dans ce secteur, c'est plutôt inquiétant.

- La pratique et la littérature nous ont appris que l'informatisation des cabinets médicaux n'est utile que dans la mesure où les processus de travail sont effectivement axés sur 
et adaptés à cette informatisation [2]. En d'autres termes, l'introduction de l'informatique dans les cabinets médicaux et les salles de consultation implique de grands changements. Quiconque minimise les modifications nécessaires pour atteindre ce but ne fait que jeter de la poudre aux yeux des médecins. Cette incursion électronique dans nos cabinets a lieu que nous le voulions ou non, mais pour ce qui est de la manière nous devons avoir notre mot à dire.

- La phrase suivante nous a frappé: «la relation entre médecins et patients, tant dans les cabinets médicaux que dans les cliniques, ne subira que de très légères modifications». Dans les cabinets médicaux, nous essayons d'appliquer le modèle des «consultations centrées sur le patient» car c'est le modèle qui respecte le mieux la relation médecinspatients et apporte les meilleurs résultats en termes de coopération. Les dossiers électroniques des patients avec banques de données et gestion du dialogue en arrière-plan, tels qu'évoqués par l'auteur, représentent en revanche une troisième instance au sein du cabinet médical. Il va de soi que ce système peut être profitable au patient, mais il exige aussi une longue et difficile procédure d'apprentissage et de prise de conscience. Les experts en informatique tiennent souvent un discours très différent qui ne répond guère à nos exigences. Même dans des pays où l'informatisation des cabinets médicaux est plus avancée, l'optimalisation des outils pour cabinets médicaux n'en est qu'à ses débuts [3].

Quelle est la quintessence de tout cela? Le développement des technologies de l'information a énormément modifié le contexte de travail non seulement dans les cliniques, mais également dans les cabinets médicaux. Nous n'en sommes cependant qu'au début, les modifications qui s'annoncent seront encore plus décisives. La carte de santé est un instrument-clé dans ce processus. Le débat sur de nombreuses questions telles que la protection des données et les coûts de la maintenance des données vient de commencer. Ni les patients, ni les groupes de professionnels concernés ne sont jusqu'ici suffisamment représentés dans ce projet.

Foundation. Electronic Medical Records: Lessons from Small Physician Practices. San Francisco: University of California; 2003.

3 de Lusignan $\mathrm{S}$. What is primary care informatics? J Am Med Inform Assoc 2003;10:304-9. ou continuer à nous en remettre aux mains des institutions, des entreprises et des assureurs?

Heinz Bhend, domaine informatique de la SSMG Marco Zoller, Association compIS

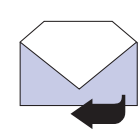

Chers confrères,

Je vous remercie pour vos contributions au sujet de l'article sur la carte de santé. Dans votre débat, vous soulevez des questions importantes et urgentes, notamment celle de savoir comment ces cartes - et surtout les concepts de traitement des données qui les sous-tendent - vont influencer l'activité médicale auprès des patients? Où trouvera-t-on les moyens d'optimaliser les processus de prise en charge? A combien se chiffre l'introduction d'une telle carte? Et, «last but not least»: à qui cela profitera-t-il?

Le Comité central s'est penché de manière différenciée sur ces questions au cours de ces derniers mois, mais pas encore dans le sens d'une prise de position fondée. Au vu de l'activisme croissant des autorités fédérales et de l'enthousiasme presque ingénu suscité par la technique notamment dans les milieux industriels (les fameux «Solution Provider»), le Comité central de la FMH a décidé qu'il est désormais temps de lancer le débat au sein du corps médical.

Sur le plan du contenu, le CC ne partage pas tous les avis exprimés, mais est d'accord avec le reproche selon lequel l'approche actuelle est insatisfaisante, à savoir une séparation floue entre la carte d'assuré en vertu de l'article 42 de la LAMal qui comporte uniquement des données administratives, et d'autres concepts plus élaborés où les données médicales des patients devraient être enregistrées - par les fournisseurs de prestations, notons-le - sur une carte de patient. Le fait que cela soit lié à d'importants investissements supplémentaires en matière d'infrastructure pour les cabinets médicaux semble évident, mais les autorités fédérales sont étonnamment muettes à ce sujet...

Toute personne qui pense qu'un simple accroissement du nombre d'ordinateurs, d'ordinateurs portables, de raccordements ADSL, de routeurs, d'interrupteurs et autres suffira à garantir l'introduction de la carte de patient dans les cabinets médicaux se trompe. Le passage à une 
documentation électronique des données des patients («dossiers médicaux électroniques») condition préalable à une carte de patient efficace et fonctionnelle comportant des contenus médicaux importants - ne représente pas seulement un défi de taille pour les médecins, mais également pour les patients, les autorités et les politiques. A ce niveau là, il ne s'agit plus d'un simple changement de support pour documenter le rapport avec les patients, mais bien d'un bouleversement important des principes fondamentaux à la base de la relation entre le médecin et son patient. Une carte de patient représente en outre un instrument de pilotage du comportement des patients.
Laissez-moi cependant préciser une chose: les changements annoncés comportent également d'importantes possibilités de développement du système de soins de santé et ce, notamment dans l'intérêt des patients. Mais rien n'est jamais gratuit et cette démarche ne peut être couronnée de succès que si toutes les personnes impliquées participent activement au débat afin de garantir que cette évolution ait lieu en toute connaissance de cause.

Ludwig T. Heuss, membre du Comité central de la FMH 